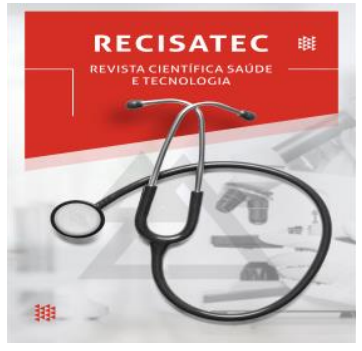

\title{
UM ESTUDO DA UTILIZAÇAO DA IMPRESSORA 3D NA ENGENHARIA E NA MEDICINA
}

\section{A STUDY OF THE USE OF THE 3D PRINTER IN ENGINEERING AND MEDICINE}

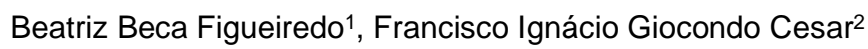

\section{RESUMO}

Com o advento da indústria 4.0 que disponibilizou novas tecnologias para as mais diversas áreas, como a impressora 3D. A prototipagem é uma ferramenta utilizada na engenharia, dentre tantas outras, para mitigar erros e otimizar processos, a qual dispõe de muitos recursos e tecnologias, dentre os quais está a prototipagem por impressão 3D, que é um processo por meio de adição de material. Esta tecnologia tem se desenvolvido de forma acelerada nos últimos anos, após as quedas de patentes e pelo desenvolvimento de novas tecnologias e materiais. Esse artigo tem como objetivo estudar a utilização da impressora 3D nas áreas, engenharia, medicina e as tecnologias envolvidas. Fundamentado em uma pesquisa bibliográfica exploratória, nas seguintes bases de dados, Google acadêmico e Web of Science, em artigos publicados a partir de 2010 sobre o tema aqui tratado. Espera-se que a partir desta pesquisa, se obtenha um maior conhecimento sobre a impressora 3D e tecnologias envolvidas.

PALAVRAS-CHAVE: Impressora 3D. Manufatura Aditiva. Aplicações da impressora 3D

\begin{abstract}
With the advent of industry 4.0 that made new technologies available for the most diverse areas, such as the $3 D$ printer. Prototyping is a tool used in engineering, among many others, to mitigate errors and optimize processes, which has many resources and technologies, among which is prototyping by $3 D$ printing, which is a process through the addition of material. This technology has been developing at an accelerated pace in recent years, following the fall of patents and the development of new technologies and materials. This article aims to study the use of 3D printer in the fields, engineering, medicine, and the technologies involved. Based on exploratory bibliographic research, in the following databases, academic google and web of Science, in articles published from 2010 on the topic dealt with here. It is expected that from this research, a greater knowledge about the $3 D$ printer and technologies involved will be obtained.
\end{abstract}

KEYWORDS: 3D Printer. Additive Manufacturing. 3D Printer Applications

\section{INTRODUCAO}

Em busca de maior competitividade no mercado, as empresas estão buscando tecnologias que as auxiliem a melhorar seus processos, a qualidade de seus produtos, a reduzir os custos e os tempos de produção.

Ao longo dos anos, em função da exigência de consumidores, as empresas foram impulsionadas a aumentar a complexidade de produtos. Em contrapartida, para se manter competitividade, os prazos para desenvolvimento tiveram de ser reduzidos. Apesar do conceito de fabricação de objetos camada-por-camada ser antigo, o início do desenvolvimento das impressoras 3D foi a partir dos anos 80. Nesta época, estas tecnologias tinham como objetivo principal, a

\footnotetext{
1 Instituto Federal de Educação, Ciência e Tecnologia de São Paulo, Campus Piracicaba

2 Instituto Federal de Educação, Ciência e Tecnologia de São Paulo, Campus Piracicaba
} 


\section{RECISATEC - REVISTA CIENTÍFICA SAÚDE E TECNOLOGIA ISSN 2763-8405}

UM ESTUDO DA UTILIZAÇAO DA IMPRESSORA 3D NA ENGENHARIA E NA MEDICINA Beatriz Beca Figueiredo, Francisco Ignácio Giocondo Cesar

construção de protótipos de maneira rápida, sem a necessidade de construção e utilização de moldes, ou mesmo de remoção de material, como em fresas e tornos. Por este motivo, a classificação destes processos de fabricação como Prototipagem Rápida teve sua difusão mais acentuada no meio industrial (CUNIDO, 2015).

A manufatura aditiva, como também é conhecida a impressão 3D, possui como seu input primordial o uso de modelos virtuais. Tais modelos virtuais são gerados via softwares computacionais do tipo Computer-Aided Design (CAD), fornecendo à impressora 3D a informação essencial para a confecção do produto desejado. A deposição e a fusão de materiais são os métodos construtivos mais regularmente utilizados por essas máquinas, as quais também variam em muito quanto ao uso de matérias-primas, isto é, diferentes tipos de polímeros, cerâmica, metais, tecidos, biomateriais, entre outros (VOLPATO, 2007).

Essa variedade de materiais servindo de base construtiva às máquinas abre caminhos para seu uso em diferentes projetos, pertencentes aos mais distintos âmbitos de atuação. Assim, a emergência da impressão 3D como um mecanismo simplificado de prototipagem rápida manifesta-se como um aliado consistente no desenvolvimento de projeto em múltiplas áreas do conhecimento, a exemplo da arquitetura, do design, da moda, das artes, saúde e educação, gerando saídas que não somente servem como protótipos ou modelos de teste, mas também como produtos plenamente funcionais e finais em muitos casos (CUNICO, 2015).

A necessidade de se fazer uma análise com relação às variáveis de impressão 3D se mostra importante para se conhecer a diversidade dessa tecnologia, e em relação às suas mais diversas áreas de atuação. Uma tecnologia que proporcionou diversas possibilidades em diferentes áreas da ciência. Esse artigo tem como objetivo estudar a utilização da impressora 3D nas áreas, engenharia, medicina e as tecnologias envolvidas. Conhecer os materiais e técnicas mais utilizadas na impressão 3D.

Neste artigo, iremos procurar responder as seguintes questões: - Quais as áreas que a tecnologia da impressão 3D é mais adotada? - Quais as tecnologias mais recentes de impressão 3D?

Para a apresentação da tecnologia de impressão tridimensional é feita, no capítulo 2, a partir das palavras-chave. No terceiro capítulo trataremos do método e metodologia da pesquisa, a sua natureza, forma de abordagem, dos objetivos e procedimentos técnicos. O quarto é abordada a teoria da pesquisa mais detalhada. O quinto apresenta os resultados sob a qual foi feita a análise da pesquisa. Por fim, no sexto e último capítulo é realizada as considerações finais.

\section{REFERENCIAL TEÓRICO}

\section{1 - MANUFATURA ADITIVA}

Os principais processos de fabricação possuem princípios baseados na moldagem do material, que envolve ou não a sua fusão (por exemplo, vários tipos de fundição de metais em moldes permanentes ou não, moldagem por injeção de plástico, metalurgia do pó, moldagem de peças em fibra de vidro etc.); na remoção (ou subtração) de material, até se chegar à forma desejada 


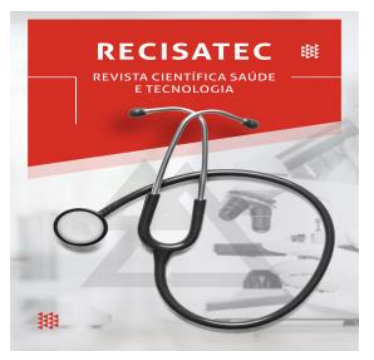

(por exemplo, torneamento, fresamento, furação, retifica, eletroerosão, usinagem química, eletroquímica etc.); na conformação, que gera a geometria final da peça a partir da deformação plástica do material inicial (por exemplo, forjamento, conformação e estampagem de chapas, extrusão, laminação, entre outros); na união de componentes (por exemplo, soldagem, brasagem, colagem, entre outros), que pode promover a junção de partes mais simples para compor uma peça mais complexa; e na divisão de componentes (por exemplo, serragem e cortes), que faz o contrário da união. No final da década de 1980, um novo princípio de fabricação baseado na adição de material (AM) foi apresentado, denominado atualmente de impressão 3D (VOLPATO, 2017).

O processo tem início com o modelo 3D da peça sendo "fatiado" eletronicamente, obtendo-se as "curvas de nível" 2D que definirão, em cada camada, onde será ou não adicionado material. A peça física é, então, gerada por meio do empilhamento (e da adesão) sequencial das camadas, iniciando na base até atingir o seu topo. De uma forma geral e mais detalhada, as etapas do processo compreendem:

a modelagem tridimensional, gerando-se um modelo geométrico 3D da peça (por exemplo, em um sistema CAD);

a obtenção do modelo geométrico 3D num formato específico para AM, geralmente representado por uma malha de triângulos, em um padrão adequado (por exemplo, STL STereoLithography, AMF - additive manufacturing format, ou outro).

o planejamento do processo para a fabricação por camada (fatiamento e definição de estruturas de suporte e estratégias de deposição de material).

a fabricação da peça no equipamento de AM; e

o pós-processamento, que varia bastante de acordo com a tecnologia (pode envolver limpeza, etapas adicionais de processamento e acabamento com processos tradicionais de usinagem por remoção).

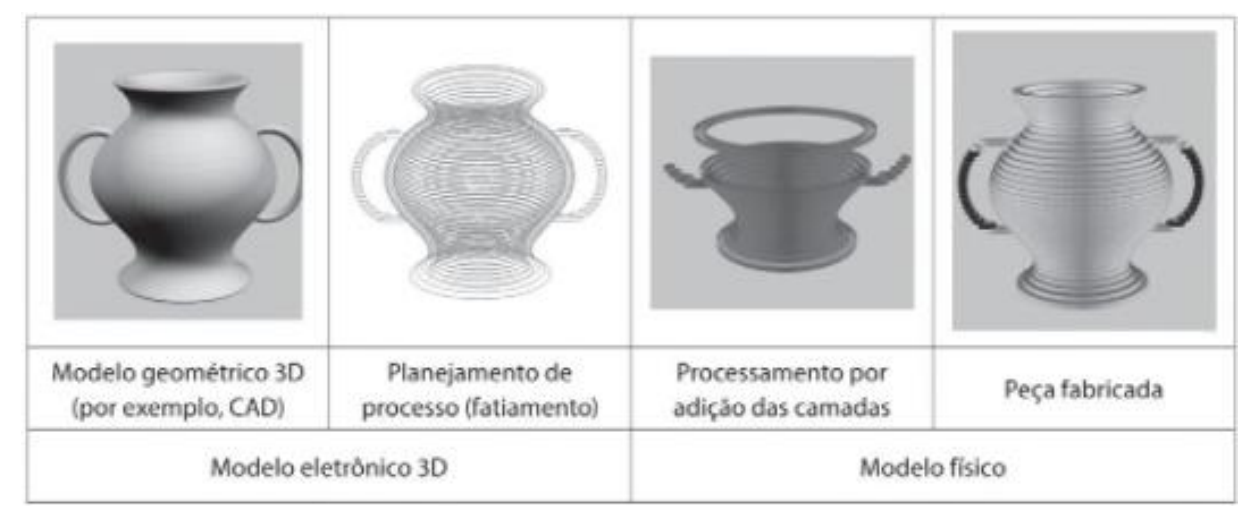

Figura 1: Representação das principais etapas do processo de AM ou impressão 3D. Fonte: Volpado (2017). 


\section{2 - IMPRESSORA 3D}

UM ESTUDO DA UTILIZAÇAO DA IMPRESSORA 3D NA ENGENHARIA E NA MEDICINA Beatriz Beca Figueiredo, Francisco Ignácio Giocondo Cesar

Entre as diversas categorias de impressoras 3D, a mais difundida e que projetou o nome da tecnologia é a impressão 3D baseada em materiais particulados. Este processo foi inicialmente desenvolvido pela Universidade Tecnológica de Massachussetts (MIT) e patenteada em 1989 por Emanuel Sachs e seus colegas pesquisadores. O princípio funcional se fundamenta na deposição de um material colante (binder) sob camada de pó cerâmico (geralmente gesso), gerando um aglomerado. Neste processo, apresentando na Figura 2, um reservatório contendo pó eleva uma plataforma enquanto um rolo distribui este pó sobre a plataforma de construção da peça (CUNIDO, 2015).

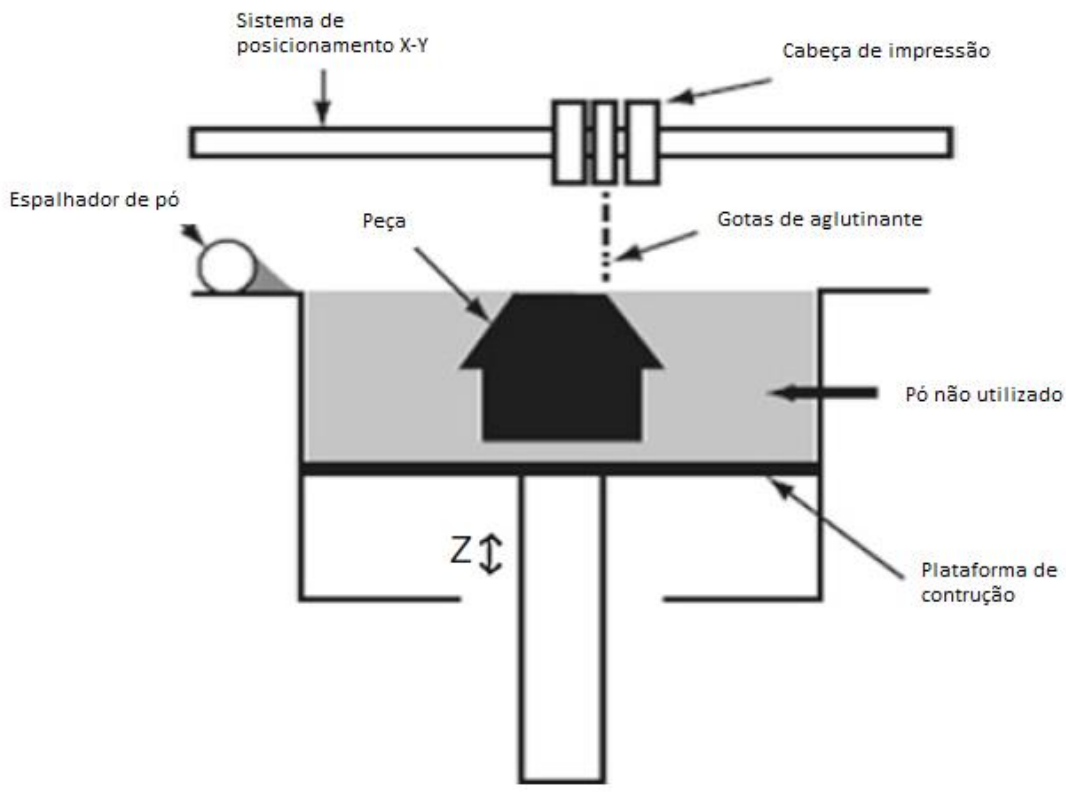

Figura 2: Ilustração esquemática do processo de impressão 3D.

Fonte: Gibson; Rosen (2010).

Em relação à espessura de deposição, esta tecnologia proporciona valores entre de 0,089 a 0,2mm, enquanto a resolução se encontra em torno de 600x540 DPI. Adicionalmente, pode-se dizer que esta precisão geral é aproximadamente $0,125 \mathrm{~mm}$. Além da velocidade de fabricação desta tecnologia chegar a 4 camadas por minuto, este processo se destaca por algumas das impressoras 3D proporcionarem objetos coloridos em até 24 bits de cores (GIBSON; ROSEN, 2010).

\section{3 - Aplicações da impressora 3D}

As impressoras 3D podem ser utilizadas em aplicações de diversas áreas tecnológicas, existem estudos voltados a área médica (BAGHERI-KHOULENJANI et al., 2019), odontológica (GJELVOLD, 2019) e construção civil (ZUO et al., 2019). Estes estudos não se limitam apenas a materiais plásticos, podendo ser usada na manufatura de alguns tipos de metais (MURR et al., 2012). 


\section{RECISATEC - REVISTA CIENTÍFICA SAÚDE E TECNOLOGIA ISSN 2763-8405}

UM ESTUDO DA UTILIZAÇAO DA IMPRESSORA 3D NA ENGENHARIA E NA MEDICINA Beatriz Beca Figueiredo, Francisco Ignácio Giocondo Cesar

Mas esta tecnologia é mais difundida entre designers, engenheiros e hobbystas. Uma impressora 3D do tipo FFF funciona depositando um material plástico em estado pastoso na forma de filamento por um percurso definido, adicionando camada por camada, formando a peça. Em relação a peças fabricadas por injeção, as manufaturadas em impressoras 3D tendem a ser menos resistentes. De acordo com Lovo et al. (2016) uma peça produzida em ABS em uma impressora 3D apresenta uma queda entre $40 \%$ e $85 \%$ (dependendo do sentido de deposição das camadas) de resistência comparado a uma peça do mesmo material produzida por um método de injeção. Por outro lado, a vantagem da impressão 3D sobre a injeção é a sua capacidade de produzir geometrias complexas, algumas vezes impossíveis nos métodos de injeção, além de ter um custo menor na fabricação quando existe apenas uma pequena demanda pela quantidade de peças.

A aplicação da impressão 3D no setor de saúde é uma das mais promissoras, tendo como exemplo na área odontológica a construção de mandíbulas (DELGADO et al., 2012), podendo oferecer soluções com melhor funcionalidade para um implante temporário ou permanente.

Outros exemplos de aplicação da impressora 3D, seriam, moldes para fabricação, utensílios domésticos, protótipos, miniaturas, carros, casas e órgãos.

\section{MÉTODO E METODOLOGIA}

Essa pesquisa teve como início uma pesquisa bibliográfica exploratória, em artigos sobre a utilização da impressora 3D em diversas áreas, buscando conhecer seus vários tipos e aplicações. Estudando sobre a novas tecnologias da impressão 3D. Desta forma, desenvolver novos conhecimentos que podem ajudar na compreensão da evolução, viabilizando seu potencial tecnológico e utilização.

\section{PESQUISA}

Assim como descrito por Dabague (2014): "A primeira técnica de se produzir um protótipo a partir de um arquivo virtual foi descoberta por Chuck Hull em 1984" e patenteada em 1986 pelo engenheiro americano Charles Hull fundador da companhia 3D Systems. Segundo Cunha (2013) inspirado na invenção das impressoras a jato de tinta, muito recente naquela época, Chuck Hull formulou o processo de estereolitografia que funcionava empregando um raio de luz ultravioleta que ao entrar em contato com um plástico ou polímero em estado líquido o solidificava, formando assim a estrutura dos objetos desejados.

Apesar de frágeis, esses objetos serviam bem ao propósito de protótipo objetivado por Chuck Hull. Esse método ainda é um dos mais utilizados atualmente, mesmo tendo sofrido modificações desde sua invenção, a arquitetura base não mudou tanto, pois segundo Aguiar e Yonezawa (2014) as impressoras mais atuais desse tipo utilizam um projetor de alta resolução, igual aos utilizados em apresentações de slides, projetando imagens sobre um recipiente repleto de resina, com uma plataforma que se movimenta verticalmente, a imagem é projetada por alguns segundos sobre a fina camada de resina causando a solidificação com o formato da imagem projetada, a plataforma com o 


\section{RECISATEC - REVISTA CIENTÍFICA SAÚDE E TECNOLOGIA} ISSN 2763-8405

UM ESTUDO DA UTILIZAÇAO DA IMPRESSORA 3D NA ENGENHARIA E NA MEDICINA Beatriz Beca Figueiredo, Francisco Ignácio Giocondo Cesar

recipiente de resina desce alguns micrômetros e o processo se repete com a projeção de uma nova imagem por cima da anterior até formar o objeto desejado. As patentes de invenção são "a criação de algo resultante da capacidade intelectual do seu autor e que representa uma solução nova para um problema existente, em uma determinada área tecnológica possuindo atividade inventiva." Dirpa (2012). A patente de invenção de Charles Hull expôs pioneiramente a tecnologia de impressão 3D para o mundo, marcando seu início até chegar ao que conhecemos atualmente. Sua patente, apesar de ter restringido o uso da estereolitografia, não impediu que novas tecnologias surgissem, como por exemplo a Modelagem por Deposição de Material Fundido (FDM) que veio a ser uma das mais conhecidas atualmente, Dabague (2014) afirma que surgiram, também, a Selective Laser Sintering (SLS) ou sinterização a laser, Selective Laser Melting (SLM) ou derretimento a laser, Polyjet e outras técnicas não difundidas amplamente. Em 1989 fora patenteada por Scott Crump a Modelagem por Deposição de Material Fundido (FDM).

O primeiro sistema de prototipagem rápida baseado na tecnologia FDM foi lançado em abril de 1992 pela empresa Stratasys (STRATASYS, 2016). Segundo Salmoria et al. (2007), essa tecnologia funciona aplicando um filamento de termoplástico de aproximadamente $1,6 \mathrm{~mm}$ de diâmetro sobre uma bandeja onde será construído o objeto, esse filamento fica armazenado em uma bobina com a ponta do mesmo presa a uma matriz de extrusão. A matriz é aquecida para fundir o termoplástico e um sistema mecânico permite que a vazão do material seja controlada sobre a bandeja na geometria requerida para o objeto. Também fora patenteada em 1989 a SLS, nesse sistema um laser é usado para fundir os grãos de pó de um material, em seguida, uma nova cobertura é adicionada dando continuidade à sinterização camada a camada. A trajetória do laser é definida por um conjunto óptico de espelhos, sendo a movimentação restrita a distribuição do particulado e ao macro posicionamento (SILVEIRA, 2015).

Tabela 1: Materiais utilizados pelas tecnologias de prototipagem rápida.

\begin{tabular}{|c|c|}
\hline Tecnologia & Materiais disponíveis \\
\hline Esteteolitografia (SLA) & Fotopolímero \\
\hline Modelagem por fusão e deposição (FDM) & Termoplásticos e metais eutéticos \\
\hline Sinterização seletiva a laser (SLS) & Termoplásticos e metais pulverizados \\
\hline Manufatura de objetos em lâminas (LOM) & Papel \\
\hline Fusão por feixe de elétrons & Ligas de titânio \\
\hline
\end{tabular}

Fonte: Raulino (2011). 
RECISATEC - REVISTA CIENTÍFICA SAÚDE E TECNOLOGIA ISSN 2763-8405

UM ESTUDO DA UTILIZAÇAO DA IMPRESSORA 3D NA ENGENHARIA E NA MEDICINA Beatriz Beca Figueiredo, Francisco Ignácio Giocondo Cesar

Tabela 2: Características da Prototipagem Rápida no Brasil.

\begin{tabular}{|l|c|c|c|c|c|}
\hline Processo / Características & SLA & SLS & LOM & 3D Print & FDM \\
\hline Variedade de Materiais & Pequena & Grande & Pequena & Média & Média \\
\hline Translucidez & Sim & Não & Não & Não & Sim \\
\hline Qualidade Superficial & Regular & Boa & Regular & Boa & Regular \\
\hline Pós-Acabamento superficial & Regular & Boa & Baixa & Boa & Regular \\
\hline Precisão & Excelente & Boa & Baixa & Boa & Regular \\
\hline $\begin{array}{l}\text { Resistência ao Impacto - } \\
\text { simulando } \\
\text { Polímeros }\end{array}$ & Regular & Boa & Baixa & Baixa & Boa \\
\hline $\begin{array}{l}\text { Resistência a Flexão - } \\
\text { simulando } \\
\text { Polímeros }\end{array}$ & Baixa & Excelente & Baixa & Baixa & Excelent \\
\hline Custo do Protótipo - no Brasil & Alto & Médio & Alto & Médio & Médio \\
\hline $\begin{array}{l}\text { Pós-Processo } \\
\text { Pós-Cura }\end{array}$ & Sim & Sim & Sim & Sim & Sim \\
\hline
\end{tabular}

Fonte: Junior (2007).

Tabela 3: Resumo das características das tecnologias de prototipagem rápida.

\begin{tabular}{|l|c|c|c|c|c|}
\hline & SLA & FDM & SLS & LOM & 3DP \\
\hline Variedade de materiais & Pequena & Média & Grande & Pequena & Média \\
\hline Qualidade superficial & Regular & Regular & Boa & Regular & Boa \\
\hline Pós-acabamento & Regular & Regular & Bom & Baixo & Bom \\
\hline Precisão & Excelente & Regular & Boa & Baixa & Regular \\
\hline Resistência ao impacto & Regular & Boa & Boa & Baixa & Baixa \\
\hline Resistência à flexão & Baixa & Excelente & Excelente & Baixa & Baixa \\
\hline Custo do Protótipo & Alto & Baixo & Alto & Alto & Médio \\
\hline Pós-cura & *Não necessita de cura quando utiliza termoplástico & Não & Não \\
\hline
\end{tabular}

Fonte: Raulino (2011).

\section{NA ENGENHARIA MECÂNICA:}

A indústria automotiva é uma das mais beneficiadas das tecnologias de prototipagem rápida. As maiores fabricantes de automóveis ao redor do mundo estão usando impressoras 3D, a fim de acelerar e melhorar o design e os processos de construção. A Ford, por exemplo, já imprimiu 500.000 


\section{RECISATEC - REVISTA CIENTÍFICA SAÚDE E TECNOLOGIA ISSN 2763-8405}

UM ESTUDO DA UTILIZAÇAO DA IMPRESSORA 3D NA ENGENHARIA E NA MEDICINA Beatriz Beca Figueiredo, Francisco Ignácio Giocondo Cesar

partes de automóveis e economizou bilhões de dólares e milhões de horas de trabalho (FORD, 2016). A tecnologia é capaz de produzir desde volantes, passando pelos assentos dos automóveis, até partes do motor dos veículos. Em 2011, a Kor Ecologic lançou o primeiro carro com toda a carroceria feita por impressão 3D, como peso de apenas $544 \mathrm{kgr}$, o que contribui para fazer uma média de $100 \mathrm{~km} / \mathrm{l}$.

A impressão 3D permite que os engenheiros verifiquem o ajuste das diferentes peças muito antes de se comprometer a produção, mostra modelos detalhados em escala, apresentando e reduzindo os custos dos projetos. Podem produzir protótipos e possuem também a capacidade de produzir peças com acabamento (TAKAGAKI, 2012).

Atualmente, todas as fábricas da Volkswagen no Brasil contam com a tecnologia da impressão 3D, estas máquinas são utilizadas pela engenharia de protótipos na confecção de peças para os projetos de veículos que serão lançados e auxilia no desenvolvimento de dispositivos utilizados na produção. As impressoras 3D materializam com a máxima precisão e sem desperdício, utilizando o material polímero, imprimindo projetos desenvolvidos em programas de desenho auxiliado por computador "CAD” (computer aided design) (CAMPOS et al., 2017).

\section{NA MEDICINA:}

A junção das duas ciências, engenharia e medicina, pode ser denominada como biônica, no qual é um campo que tem o estudo dos implantes sintéticos em sistemas naturais como por exemplo, próteses e órgãos artificiais. Nesse caso, a engenharia enxerga o corpo humano como uma máquina biológica. Dessa forma, a engenharia dedica-se em buscar meios de substituição das funções biológicas pela tecnologia. Muitos pesquisadores têm buscado não apenas produções de próteses, mas também produções de tecidos cardíacos, células tronco, cartilagem, ossos entre outros (TOMÉ, 2015).

As próteses desenvolvidas de forma individual aumentam em $40 \%$ a velocidade de regeneração do tecido, além de serem construídos na impressora 3D vasos sanguíneos, redes vasculares, ataduras, ossos, orelhas, narizes, olhos, próteses dentárias. O maior avanço e benefício esperado da impressora 3D para a área da saúde será o desenvolvimento de órteses de órgãos humanos. A impressão 3D também tem sido amplamente utilizada na Tecnologia Assistiva (TA). A alta complexidade individual anatômica, cinesiologia e biomecânica deve ser considerada ao se pensar em um recurso de Tecnologia Assistiva, além das peculiaridades de cada caso. Não obstante da alta complexidade dos equipamentos de TA, o uso da impressora 3D para a confecção de pacientes com disfunções físicas é algo transformador.

As possibilidades trazidas através deste recurso vão desde o refinamento das adaptações de forma individual, à extinção de uso de alguns materiais, que podem causar incômodos ou intercorrências, uma vez que podem permitir um ajuste perfeito, sem sobreposições ou costuras (bastante comuns em adaptações de termoplásticos), além da melhora da estética do produto e do 
menor custo em relação as adaptações já existentes no mercado (CRUZ; JÚNIOR; SARMANHO, 2018).

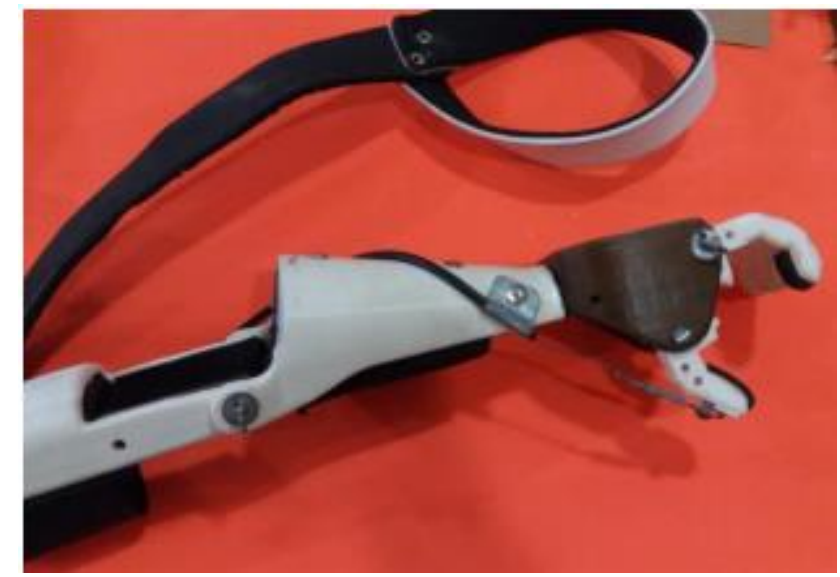

Figura 3: Prótese transradial mecânica esquerda.

Fonte: Cruz et al. (2018).

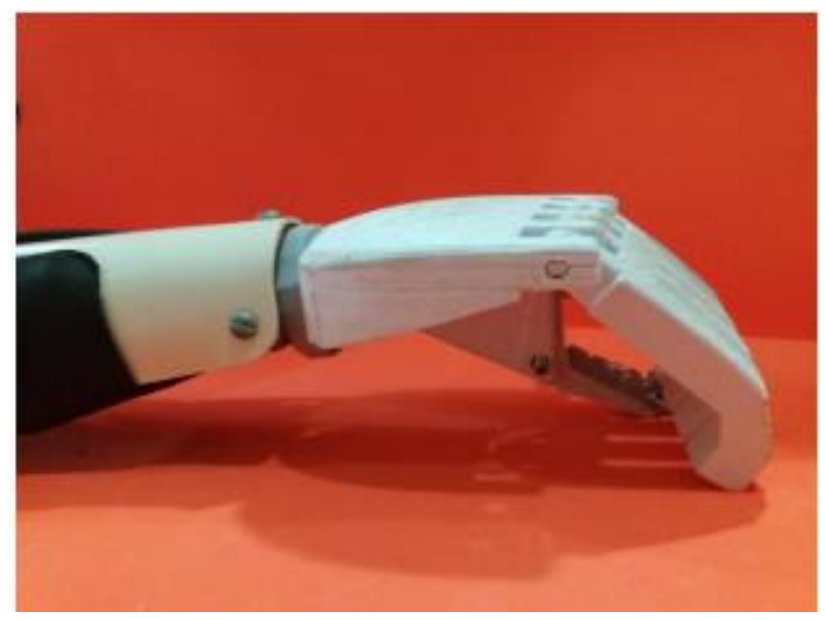

Figura 4: Prótese para amputação parcial de mão com acionamento a partir da extensão de punho.

Fonte: Cruz et al. (2018).

O princípio da impressora 3D é o mesmo da convencional, só que no lugar de tinta, cientistas introduzem no aparelho pó, gel ou filamento de metal ou de plástico, que, no lugar de letras, imprime camada por camada de peças tridimensionais desde dedos, crânios, peles até células tronco embrionárias - os estudiosos deram a isso o nome de bioprinting (PALMA; KLAPER, 2015; MATOZINHOS et al., 2017).

A impressora 3D tem viabilizado não só a elaboração de novas abordagens terapêuticas, mas também a dinamização de procedimentos cirúrgicos, garantindo um melhor prognóstico e um incremento na qualidade de vida dos pacientes (BERNARDES, 2018). Por isso, muitos cirurgiões 


\section{RECISATEC - REVISTA CIENTÍFICA SAÚDE E TECNOLOGIA ISSN 2763-8405}

UM ESTUDO DA UTILIZAÇAO DA IMPRESSORA 3D NA ENGENHARIA E NA MEDICINA Beatriz Beca Figueiredo, Francisco Ignácio Giocondo Cesar

ortopédicos viram na possibilidade de criar próteses e órteses customizadas, respeitando as regiões de amputação, os segmentos preservados e a mobilidade restante, uma forma de devolver a autonomia àqueles que a tiveram abalada (MALLMANN, 2018).

Um exemplo de utilização dessa técnica no Brasil é pelo neurocirurgião Dr. Joel Teixeira, dos grupos de dor e de coluna do Hospital das Clínicas e do Hospital Alemão Oswaldo Cruz, em São Paulo, no qual ele cria protótipos de colunas a partir de tomografias computadorizadas dos seus pacientes, permitindo que seja um tratamento personalizado, atendendo as demandas e propiciando o que a medicina busca desde seus primórdios: a qualidade de vida.

Outro caso com resultados impressionantes envolveu uma criança de 3 anos na China, que apresentava hidrocefalia. A impressora foi utilizada para criar um "crânio artificial", o qual permitiu após 17 horas de cirurgia substituir a calota craniana que havia sido edemaciada pelo acúmulo de líquido (PALMA; KLAPPER, 2015).

A cirurgia plástica, por sua vez, foi uma área que se beneficiou de inúmeras inovações tecnológicas ao longo de sua evolução médica, tanto no que tange a técnicas, quanto à instrumentos. Por trabalhar essencialmente com o maior tecido do corpo, a pele, requer cuidados ainda maiores na tentativa de replicar partes dela por meio da impressão 3D (BERNARDES, 2018). No entanto, apesar de todos esses avanços, a prototipagem rápida ainda não é parte da realidade da maioria dos grandes centros espalhados pelo mundo, uma vez que a medicina ainda esbarra na heterogeneidade tanto no que tange ao desenvolvimento dos países, quanto aos recursos disponíveis nessas regiões (GUERRA NETO, 2018).

Assim, mesmo com os avanços recentes, o uso dessa tecnologia ainda enfrenta muitas dificuldades, uma das principais é o custo relativamente elevado dos modelos capazes de combinar diferentes matérias primas para a produção de peças mais resistentes, o que faz que seu uso ainda se restrinja aos países desenvolvidos (BERNARDES, 2018). Além do custo, outro fator limitante diz respeito ao tempo necessário para a produção e acurácia do modelo gerado.

Em um grande estudo realizado por Li et al. (2016), foi relatado que o tempo necessário oscilou entre 10h e 2 semanas, o que dificulta o emprego na emergência, haja vista que os quadros que ali estão demandam ações imediatas (GUERRA NETO, 2018). A fim de diminuir as barreiras a serem enfrentadas, é preciso difundir o conhecimento a respeito de tal tecnologia para que mais pessoas na área da saúde saibam como utilizá-la nos variados casos encontrados nos hospitais. Ainda, informações sobre abordagens inovadoras, como essa, podem impulsionar novas pesquisas e descobertas relacionadas à área contribuindo para avanços adicionais no campo da medicina (BERNARDES, 2018).

\section{NA CONSTRUÇÃO CIVIL:}

A construção habitacional foi identificada como uma área que poderia se beneficiar da aplicação de impressão em 3D. Como cada edifício é único, requer um investimento significante em ferramentas especializadas. Inversamente, uma solução de impressão 3D exige apenas uma 

ISSN 2763-8405

UM ESTUDO DA UTILIZAÇO DA IMPRESSORA 3D NA ENGENHARIA E NA MEDICINA Beatriz Beca Figueiredo, Francisco Ignácio Giocondo Cesar

mudança no modelo de computador. Dessa forma, espera-se proporcionar melhorias no custo, eficiência, e velocidade para o consumidor e para o contratante (GARDNER et al., 2013).

A construção civil tem acompanhado os desenvolvimentos em técnicas de impressão 3D e começou a aplicá-los em maior escala. Principalmente a impressão de concreto e materiais cimentícios ultimamente tem ganhado muito interessados no campo da arquitetura e construção. A primeira técnica de impressão 3D para construção civil é chamada de Contour Crafting, que é uma tecnologia de fabricação aditiva que usa o controle de computador para formar estruturas de materiais cerâmicos e a base de cimento (WOLFES, 2015).

Além da Contour Crafting, há vários grupos de pesquisa que utilizam misturas especiais a base de cimento que podem ser impressos, formando grandes partes que podem ser adequados para utilização como elementos de construção. Um grupo da Universidade de Loughborough desenvolveu em 2008 um sistema baseado na extrusão mais tradicional que eles chamam de Concrete Printing que imprime partes a base de cimento. Sua abordagem é muito semelhante ao da operação de qualquer outra máquina FDM, no entanto, eles têm produzido peças grandes com todos os tipos de formas, com a possibilidade adicional de elementos internos, tais como a água, de gás ou de eletricidade. Já a técnica chamada de D-Shape, criada por Enrico Dini, utiliza um processo de deposição de pó (GARDNER et al., 2013).

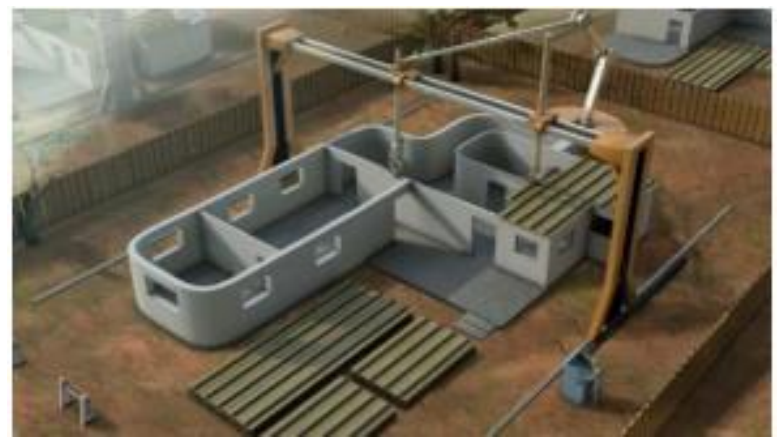

Figura 5: Esquema de construção com Contour Crafting. Fonte: Contour Crafting (2016).
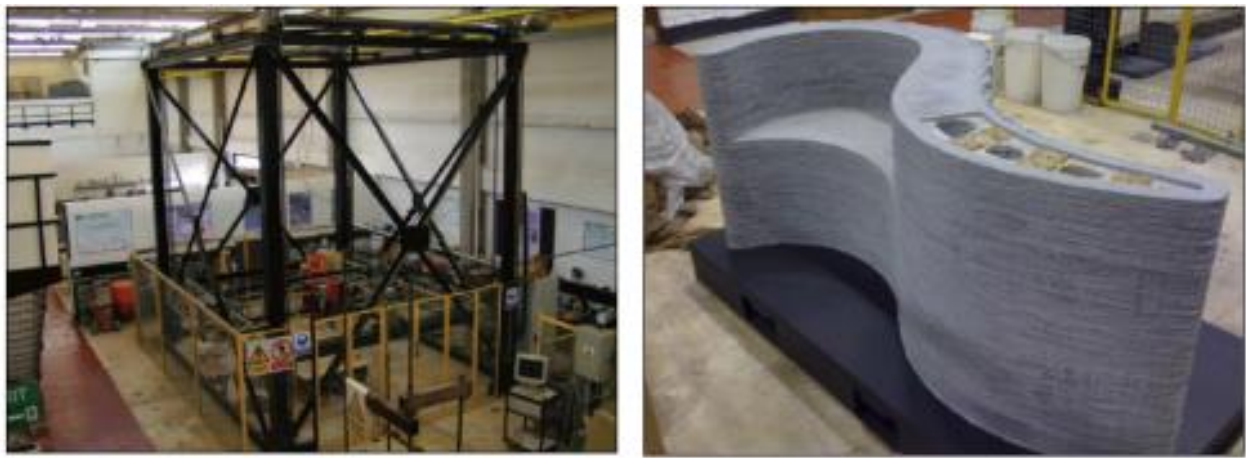

Figura 6: Concrete Printing. Fonte: Lim et al., (2012). 


\section{RECISATEC - REVISTA CIENTÍFICA SAÚDE E TECNOLOGIA} ISSN 2763-8405

UM ESTUDO DA UTILIZAÇAO DA IMPRESSORA 3D NA ENGENHARIA E NA MEDICINA Beatriz Beca Figueiredo, Francisco Ignácio Giocondo Cesar

\section{ANÁLISE DOS RESULTADOS}

Após serem feitas pesquisas e estudos, esperou-se mostrar o quanto a impressora 3D é um salto da tecnologia e o quanto irá afetar de forma positiva o mercado tecnológico.

O funcionamento das impressoras 3D é bastante similar ao das impressoras comuns usadas no dia a dia, sendo que suas diferenças dizem respeito apenas ao tipo de material ejetado e ao motor, que conta com um a mais para controlar a altura do produto.

Atualmente já é possível imprimir órgãos humanos com células vivas. Na medicina, próteses dentárias e aparelhos ortodônticos são feitos a partir de um molde da boca do paciente. Mandíbulas inteiras foram substituídas por próteses impressas em titânio. Objetos impressos são utilizados para prototipagem em praticamente todos os tipos de dispositivos. Nas áreas da arquitetura e engenharia civil, as impressoras estão sendo muito utilizadas para construir maquetes de casas e prédios. Pensando no futuro da impressão 3D, profissionais estudam meios para desenvolver uma impressora capaz de construir prédios de concreto, utilizando o mesmo princípio das impressoras atuais, mas em uma escala muito maior. Ao mesmo tempo, pesquisadores trabalham para desenvolver uma impressora 3D em nível molecular. A expectativa para o futuro dessas impressoras é que elas tragam mudanças revolucionárias equivalentes às mudanças ocorridas pelo surgimento da produção em massa.

Existem diferentes técnicas para a prototipagem rápida. Algumas impressoras extrudam plástico derretido em camadas para fazer os objetos (FDM), enquanto outras usam laser para endurecer camadas de resina (SLA) ou pó (SLS). Essas tecnologias vêm apresentando bons resultados em diversos setores como na marinha, na produção de peças personalizadas em navios, e na aviação, contribuindo para a diminuição do peso dos aviões, o que resulta na redução do consumo de combustível e na emissão de poluentes no ar. A medicina é outra área de grandes avanços, com a impressão de réplicas de órgãos, e com as próteses ortopédicas impressas, que além de se adaptarem as características físicas de seus usuários apresentam um preço competitivo.

A criação e patenteamento das tecnologias SLA, SLS e FDM, permitiram que essas técnicas fossem estudadas sob um amparo legal, fortalecendo as pesquisas sobre as mesmas. A inserção da impressão 3D na área da saúde marcou o avanço dessa tecnologia em outras áreas afins. A impressão 3D tem sido inserida fortemente em praticamente todas as áreas em que se mostra útil, à medida que se torna possível utilizar novos materiais, surgem automaticamente novas aplicações para estes.

\section{CONSIDERAÇÕES FINAIS}

A tecnologia impressão 3D pode revolucionar a indústria como um todo, de forma que ela reduz os custos para a produção individualizada e para a customização. As impressoras possuem tecnologia que gera poucos resíduos, exige menos mão de obra comparada a outros processos construtivos e facilita a criação de protótipos. 


\section{RECISATEC - REVISTA CIENTÍFICA SAÚDE E TECNOLOGIA ISSN 2763-8405}

UM ESTUDO DA UTILIZAÇAO DA IMPRESSORA 3D NA ENGENHARIA E NA MEDICINA Beatriz Beca Figueiredo, Francisco Ignácio Giocondo Cesar

As pesquisas e inovações realizadas na área de fabricação aditiva (impressoras 3D), pode-se indicar que teve um tremendo avanço nos últimos anos. Com isto, diversas áreas puderam ser beneficiadas e foi possível identificar o grande potencial tecnológico desta tecnologia.

Uma das formas mais evidentes de observar este aumento de pesquisas relacionadas a impressoras 3D e manufatura aditiva é através do número de patentes geradas ao longo do tempo. Isto indica que além das impressoras 3D terem um grande índice de aceitação no mercado, as empresas, de um modo geral, preveem um potencial de aplicação muito alto. Logo, o grande número de patentes geradas nos últimos anos pode também ser fruto de um investimento pesado relacionado aos processos de manufatura aditiva.

Por fim, espera-se poder entender melhor como funciona essa tecnologia que promete facilitar e viabilizar o uso de protótipos e ferramentas nas necessidades dos consumidores.

\section{REFERÊNCIAS}

AGUIAR, L. C. D.; WILSON, M. Y. Construção de instrumentos didáticos com impressoras 3d. [S. I: S. n.], 2014 Disponível em: http://sinect.com.br/anais2014/anais2014/artigos/tic-no-ensinoaprendizagem-de-ciencias-e-tecnologia/01409583389.pdf. Acesso em: 07 ago. 2021.

BERNARDES, J. Pele impressa em 3D substitui animais em teste de cosméticos. Jornal da USP, São Paulo, p. 1-1, 15 jan. 2018. Disponível em: https://jornal.usp.br/ciencias/pele-impressa-em-3dsubstitui-animais-em-teste-de-cosmeticos/. Acesso em: 04 ago. 2021.

CAMPOS, M. M.; MENDES, C. R.; SIEMON, F. B. Estudos de caso da indústria 4.0 aplicados em uma empresa automobilística. POSGERE, v. 1, n. 4, p. 15-25, set. 2017. ISSN 2526-4982. Disponível em:

https://www.researchgate.net/profile/CleitonMendes/publication/321152959 ESTUDOS DE CASO DA INDUSTRIA 40 APLICADOS EM UM A EMPRESA AUTOMOBILISTICA/links/5a10da610f7e9bd1b2bf331d/ESTUDOS-DE-CASO-DAINDUSTRIA-40-APLICADOS-EM-UMA-EMPRESA-AUTOMOBILISTICA.pdf. Acesso em: 16 ago. 2021.

CONTOUR CRAFTING. 2016. Disponível em: http://www.contourcrafting.org. Acesso em: 04 ago. 2021.

CRUZ, L. M. S.; JÚNIOR, J. L. R.; SARMANHO, A. P. S. Impressora 3d no desenvolvimento de pesquisas com próteses. Revisbrat, v. 2, n. 2, 2018. Disponível em: https://revistas.ufri.br/index.php/ribto/article/viewFile/15022/pdf. Acesso em: 04 ago. 2021.

CUNHA, H. A. Impressoras 3D: o direito da propriedade intelectual precisará alcançar novas dimensões?. 2013. TCC (Graduação) - FGV, Rio de Janeiro, 2013. Disponível em: http://hdl.handle.net/10438/12642. Acesso em: 07 ago. 2021.

CUNICO, M. W. M. Impressora 3D: o novo meio produtivo. Curitiba: Concep3D Pesquisas $\begin{array}{lcc}\text { Cientificas } & \text { Ltda, } & \text { Disponível } \\ \text { https://play.google.com/books/reader?id=CybwCQAAQBAJ\&pg=GBS.PP1\&hl=pt- }\end{array}$

BR\&Ir=\&printsec=frontcover. Acesso em: 04 ago. 2021.

DABAGUE, L. A. M. O processo de inovação no segmento de impressoras 3d. 2014. Monografia (Graduação) - Universidade Federal do Paraná, Curitiba, PR, 2014. Disponível em: 


\section{RECISATEC - REVISTA CIENTÍFICA SAÚDE E TECNOLOGIA ISSN 2763-8405}

UM ESTUDO DA UTILIZAÇAO DA IMPRESSORA 3D NA ENGENHARIA E NA MEDICINA Beatriz Beca Figueiredo, Francisco Ignácio Giocondo Cesar

https://acervodigital.ufpr.br/bitstream/handle/1884/37115/MONOGRAFIA19-2014-

1.pdf?sequence=1\&isAllowed=y. Acesso em: 04 ago. 2021.

DELGADO, J.; LAURETTI, C. A. R.; CAMILO, A. A.; SILVA, J. V. L.; SERENO, L.; CIURANA, J. Mandible Reconstruction Using an Additive Manufacturing Technology. In: Anais... 1st International Conference on Design and Processes for Medical Devices, Brescia. 1st International Conference on Design and Processes for Medical Devices PROMED. Rivoli: Neos Edizione, 2012. v. 1. p. 275-278.

DIRPA - Diretoria de patentes. Diretrizes de exame de patente de modelo de utilidade. Brasília: INPI Instituto Nacional da Propriedade Industrial, 2012. Disponível em: http://www.inpi.gov.br/legislacao-arquivo/docs/resolucao 85-13-anexo diretrizes mu.pdf. Acesso em: 07 ago. 2021.

FORD. Building in the Automotive Sandbox. [S. I.]: Ford, [S. d.] Disponível em: https://corporate.ford.com/innovation/building-in-the-automotive-sandbox.html. Acesso em: 16 ago. 2021.

GARDNER, M.; ALWI, A.; KARAYIANNIS, S. et al. Construktion, MegaScale 3D Printing. Reino Unido: University of Surrey, 2013.

GIBSON, I.; ROSEN, D. et al. Additive Manufacturing Technologies: Rapid Prototyping to Direct Digital Manufacturing. [S. l.]: Springer, 2010. Disponível em: https://www.emerald.com/insight/content/doi/10.1108/aa.2012.03332baa.010/full/html. Acesso em: 06 ago. 2021.

GJELVOLD, B. Accuracy of surgical guides from 2 different desktop 3D printers for computed tomography-guided surgery. Malmö, Suécia: Centre of Dental Specialist Care, Lund, e Department of Prosthodontics, Faculty of Odontology, University of Malmö, 2019.

GUERRA NETO, C. L. de B. et al. Tecnologia 3D na saúde: Uma visão sobre órteses e próteses, tecnologias assistivas e modelagem 3D. Rio Grande do Norte: Sedisufrn, 2018. 95 p.

LIM, S.; BUSWELL, R.; LE, T. et al. Developments in construction-scale additive manufacturing processes. Automation in Construction, v. 21, p. 262-268, 2012.

LOVO, J. F. P.; FORTLULAN, C. A. Estudo de propriedades mecânicas e anisotropia em peças fabricadas por manufatura aditiva tipo fdm. In.: 1 SiPGEM - 1o Simpósio do Programa de PósGraduação em Engenharia Mecânica. Escola de Engenharia de São Carlos - Universidade de São Paulo. São Carlos - SP, 12 e 13 de setembro de 2016.

MALLMANN, T. da S. O uso de impressão 3D no auxílio às pessoas usuárias de órteses: um projeto de design focado em tecnologia assistiva. 2018. 112 f. Monografia (Especialização em Design) - Univates, Lajeado, 2018.

MATOZINHOS, I. P. et al. Impressão 3D: Inovações no campo da medicina. Revista Interdisciplinar Ciências Médicas-MG, p.143-162, 2017.

MURR, L. E.; MARTINEZ, E.; AMATO, K. N.; GAYTAN, S. M.; HERNANDEZ, J.; RAMIREZ, D. A.; WICKER, R. B. Fabrication of Metal and Alloy Components by Additive Manufacturing: Examples of 3D Materials Science. Journal of Materials Research and Technology, v. 1, n. 1, p. 42-54, 2012.

PALMA, F. C.; KAPPLER, A. F. O que é e como funciona uma impressora 3D. [S. I.: S. n.], 2015. Disponível em: www.abrifar.org.br/novo/Site/anexos/Boletim ABRIFAR 030 que é e como funcio na uma impressora 3D.pdf. Acesso em: 04 ago. 2021. 


\section{RECISATEC - REVISTA CIENTÍFICA SAÚDE E TECNOLOGIA ISSN 2763-8405}

UM ESTUDO DA UTILIZAÇAO DA IMPRESSORA 3D NA ENGENHARIA E NA MEDICINA Beatriz Beca Figueiredo, Francisco Ignácio Giocondo Cesar

RAULINO, B. R. Manufatura aditiva: desenvolvimento de uma máquina de prototipagem rápida baseada na tecnologia fdm (modelagem por fusão e deposição). TCC (Graduação) - Universidade de Brasília, Brasília, 2011. Disponível em: http://alvarestech.com/temp/PrototipagemRapida/Relat\%F3rio\%20TG2\%20\%20Bruno\%20Ribeiro.pdf. Acesso em: 10 ago. 2021.

SALMORIA, G. V.; CARDENUTO, M. R.; AHRENS, C. H.; LAFRATTA F. Prototipagem rápida por impressão $3 \mathrm{~d}$ com resinas foto curáveis: uma análise sobre as tecnologias disponíveis no mercado nacional. In.: Anais... 9ำ Congresso Brasileiro de Polímeros. 2007. Disponível em: https://www.ipen.br/biblioteca/cd/cbpol/2007/PDF/79.pdf. Acesso em: 09 ago. 2021.

SELHORST JUNIOR, A.; CANCIGLIERI JUNIOR, O.; IAROZINSKI NETO, A. Análise comparativa entre os processos de prototipagem rápida por deposição ou remoção de material na concepção de novos produtos. In.: Anais... ABCM, 2007. Disponível em: http://www.abcm.org.br/anais/cobef/2007/files/121001084.pdf. Acesso em: 10 ago. 2021.

SILVEIRA, C. A. Integração de um sistema de impressão 3d em uma arquitetura modular de posicionamento cartesiano. 2015. Dissertação (Mestrado) - Universidade Federal de Santa Catarina, Centro Tecnológico, Programa de Pós-Graduação em Engenharia Mecânica, Florianópolis, 2015 Disponível em: https://repositorio.ufsc.br/handle/123456789/157339. Acesso em: 09 ago. 2021.

TAKAGAKI, L. K. Tecnologia de impressão 3D. Revista Inovação Tecnológica, v. 2, n. 2, p. 28-40, jul./dez. 2012.

TOMÉ, H. É. Novo conceito para salvar vidas: engenharia e medicina, ciências que juntas melhoram, transformam e salvam vidas utilizando a impressoras 3D. 2015. TCC (Graduação) Centro Universitário do Sul de Minas, Varginha, 2015. Disponível em: http://repositorio.unis.edu.br/handle/prefix/1089. Acesso em: 04 ago. 2021.

VOLPATO, N. Prototipagem Rápida: Tecnologias e Aplicações. São Paulo: Blucher, 2007. Disponível em: https://books.google.com.br/books?hl=ptBR\&|r=\&id=10O6DwAAQBAJ\&oi=fnd\&pg=PA1\&dq=Prototipagem + R\%C3\%A1pida $:+$ Tecnologias $+e+A$ plica\%C3\%A7\%C3\%B5es\&ots=3UgllJMgzQ\&sig=SxZatxskzmCMJaSJmWAucvXxUQ8\#v=onepage\& q=Prototipagem\%20R\%C3\%A1 pida\%3A\%20Tecnologias\%20e\%20Aplica\%C3\%A7\%C3\%B5es\&f=fal se. Acesso em: 06 ago. 2021.

WOLFES, R. 3D Printing of Concrete Structures. Graduation (Thesis) - Eindhoven University of Technology, Eindhoven, 2015.

ZAHRA, Z. T.; SHADAB, B. K.; HAMID, M.; SAEID, A. 3D in vitro cancerous tumor models: using 3D printers. Iran: Department of Polymer and Color Engineering, Amirkabir University of Technology, e Cancer Biology Research Center, Tehran University of Medical Sciences, 2019. 